\title{
Pathophysiology of Plasmin System
}

\section{Some Evidence Suggesting an Entity of Enzyme Multiplicity in the Euglobulin Fraction}

\author{
By
}

\author{
Masahiro Maki and Kazuma Nagasawa \\ From the Department of Obstetrics and Gynecology, Faculty of Medicine, \\ Hirosaki University, Hirosaki; Director: Prof. S. Sh inagawa \\ (Received for publication, July 15, 1963)
}

It has been often noted that the spontaneous fibrinolytic, fibrinogenolytic, caseinolytic and TAMe ( $\mathrm{p}$-Toluenesulfonyl-L-Arginine Methylester)-lytic activities of the euglobulin fraction are not always parallel with one another in clinical tests of the same patients plasma. If these activities result from one enzyme, the activity values should be definitely proportional regardless of the difference of plasma sample. Therefore, it may be presumed that there are two or more enzymes of a similar chemical nature in the euglobulin fraction. Okamoto and his co-workers ${ }^{1)}$ observed, independently from our study, facts supporting the hypothesis of the multiplicity of proteolytic enzymes from the standpoint of the varying behavior of the enzyme for their specific inhibitors.

In this paper, the evidence is further described from the results of clinica and experimental observations.

\section{MATERIALS AND METHODS}

1. Spontaneous enzyme activity of the euglobulin fraction

The spontaneous (without the use of artificial activating procedure) fibrinolytic, caseinolytic and TAMe-lytic activities of the euglobulin fraction were determined on surgical and obstetrical patients by the method previously described in the series of this study. ${ }^{2}$ )

2. Fractionation of the euglobulin on DEAE (Diethylaminoethyl)-cellulose column chromatograph

\section{$i$. Separation of euglobulin fraction}

The euglobulin fraction was obtained by adding $950 \mathrm{ml}$. of acetic acid water (a mixture of 0.32 volume of $1 \%$ acetic acid and 19 volumes of distilled water) to

真木正博，長沢一磨 
$50 \mathrm{ml}$. of citrated plasma. The precipitated euglobulin fraction was collected by centrifuging, and then dissolved in $20 \mathrm{ml}$. of potassium phosphate buffer ( $\mathrm{pH}$ $7.8,0.0175 \mathrm{M})$.

\section{ii. Column chromatography on DEAE-cellulose}

DEAE-cellulose of $0.93 \mathrm{meq} / \mathrm{g}$, an anion exchange adsorbent, was obtained as a dry powder from the Brown Co. The powder was treated with $1 \mathrm{~N}$ sodium hydroxide to obtain a suspension of uniform particles, following which it was washed with large volume of distilled water and then equilibrated with starting buffer. The suspension of equilibrated adsorbent was poured gently onto the column of $2 \times 30 \mathrm{~cm}$, and allowed to settle under gravity to the height of $20 \mathrm{~cm}$. Then the adsorbent was washed with $100 \mathrm{ml}$. of the starting buffer. The above obtained euglobulin solution, $10 \mathrm{ml}$., was pipetted gently onto the resin, and the walls of the column were washed twice with $10 \mathrm{ml}$. of the starting buffer. The chromatograph was developed by the step-wise elution with $100 \mathrm{ml}$. of four different kinds of each solvent (Table I) in the order of increasing molar concentration and simultaneously decreasing $\mathrm{pH}$ value. Fractions divided into $10 \mathrm{ml}$, were collected at a flow rate of about $1 \mathrm{ml}$. per minute.

TABLE I. Solvents Used for Eluation

\begin{tabular}{l|lll}
\hline & \multicolumn{1}{|c}{ Solvents } & pH & Molar conc. \\
\hline Starting buffer & Potassium phosphate buffer & 7.80 & 0.0175 \\
Second buffer & Potassium phosphate buffer & 7.16 & 0.04 \\
Third buffer & Potassium phosphate buffer & 6.64 & 0.1 \\
Final solvent & 0.0025 M hydrochloric acid & & \\
& containing 1 M sodium chloride & &
\end{tabular}

\section{iii. Determination of protein concentration}

Protein concentration was determined on $0.4 \mathrm{ml}$. aliquot of the eluates as tyrosine by the phenol reagent of Folin Ciocalteu. When turbidity occurred in the reaction mixture, the optical density was measured after centrifugation.

\section{iv. Caseinolytic activity}

The caseinolytic activity of streptokinase (SK)-activatable proteolytic enzyme in the eluates was determined by incubating a mixture of $0.4 \mathrm{ml}$. aliquot of the eluates, $0.1 \mathrm{ml}$. of SK (500 units, Varidase from Lederle Co. was used as a SK source) and $2.5 \mathrm{ml}$. of $2 \%$ casein at $37^{\circ} \mathrm{C}$ for two hours. Then, the reaction was stopped by adding $2.5 \mathrm{ml}$. of $0.44 \mathrm{M}$ trichloroacetic acid (TCA). The activity was measured by reading optical density of the developed color with phenol reagent against the blank. The blank was prepared in the same way by adding $0.1 \mathrm{ml}$. of saline solution instead of SK.

\section{v. TAMe-lytic activity}

The TAMe-lytic activity of SK-activatable esterolytic activity in the eluates determined by an essentially similar method of Troll et al. ${ }^{3)}$ To the test tube, 
$0.5 \mathrm{ml}$. of $0.1 \mathrm{M}$ TAMe, $1.25 \mathrm{ml}$. of $0.5 \mathrm{M}$ tris buffer $(\mathrm{pH}, 9.0)$ and $0.25 \mathrm{ml}$. of SK (1,000 units) were added and incubated at $37^{\circ} \mathrm{C}$ for 5 minutes. Then, $0.5 \mathrm{ml}$. aliquot of the eluates was added to the above reaction mixture, and further incubated at $37^{\circ} \mathrm{C}$ for 60 minutes. To the resultant $1 \mathrm{ml}$. of the mixture, $2.5 \mathrm{ml}$. of $37 \%$ formalin (previously adjusted to $\mathrm{pH} 8.0$ with sodium hydroxide) and 0.2 $\mathrm{ml}$. of $0.01 \%$ phenol red were added. The mixture was titrated with $0.05 \mathrm{M}$ sodium hydroxide. The blank was run simultaneously in the same by adding saline solution instead of SK. The TAMe-lytic activity was arbitrarily expressed by the difference of the amounts in $\mathrm{ml}$. of $0.05 \mathrm{~N}$ sodium hydroxide required to neutralize the test and the blank.

vi. Fibrinolytic activity

The fibrinolytic activity of SK-activatable proteolytic enzyme in the eluates was measured by the lysis time of a fibrin clot method. To the test tube containing $0.2 \mathrm{ml}$. of the eluates, $0.1 \mathrm{ml}$. of SK (100 units), $0.1 \mathrm{ml}$. of thrombin (10 $\mathrm{NIH}$ units) and $1 \mathrm{ml}$. of $0.1 \%$ fibrinogen were added and incubated for at $37^{\circ} \mathrm{C}$. The fibrinolytic activity was expressed by the time, in minutes, required for complete lysis of the fibrin clot.

vii. Antitrypsin activity

The antitrypsin activity of the eluates was determined on $0.5 \mathrm{ml}$. of the eluates by the previously described method of Maki. ${ }^{2}$ The inhibitory activity was simply expressed by the difference of optical density at $275 \mathrm{~m} \mu$ between the standard and the test compared to the blank.

\section{RESULTS AND DISCUSSION}

1. Some evidence suggesting an entity of enzyme multiplicity from the aspect of clinical observations

The spontaneous fibrinolytic, fibrinogenolytic and caseinolytic activities were determined on the same source of euglobulin fraction obtained from healthy and diseased adults at random. The relation between spontaneous fibrinolytic and fibrinogenolytic activities was statistically highly significant with only 3 exceptions in 61 cases (Fig. 1). In general, the activity of fibrinolysis was 10-20 $\%$ higher than that of fibrinogenolysis. The reversed situation was often observed in cases of hypofibrinogenemia.

The relation between spontaneous fibrinolysis and caseinolysis of the euglobulin fraction was statistically significant also, but the correlation was weak (Fig. 2).

In the following observations, the spontaneous fibrinolytic, caseinolytic and TAMe-lytic activities were determined on the same source of euglobulin fraction obtained from patients with surgical operations or delivery. The results are shown in Figs. 3 and 4. In the patients with surgical operations, a marked elevation of 


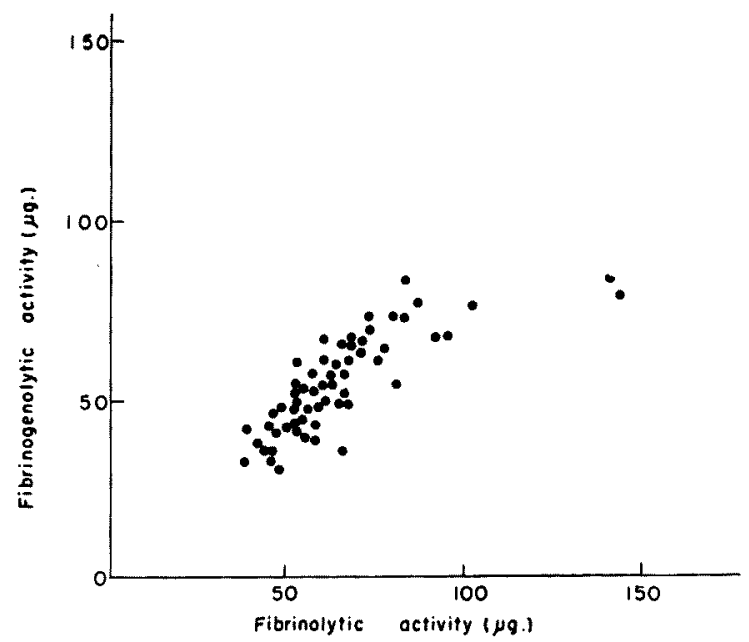

Fig. 1. Relation between spontaneous fibrinolytic and fibrinogenolytic activities of euglobulin fraction.

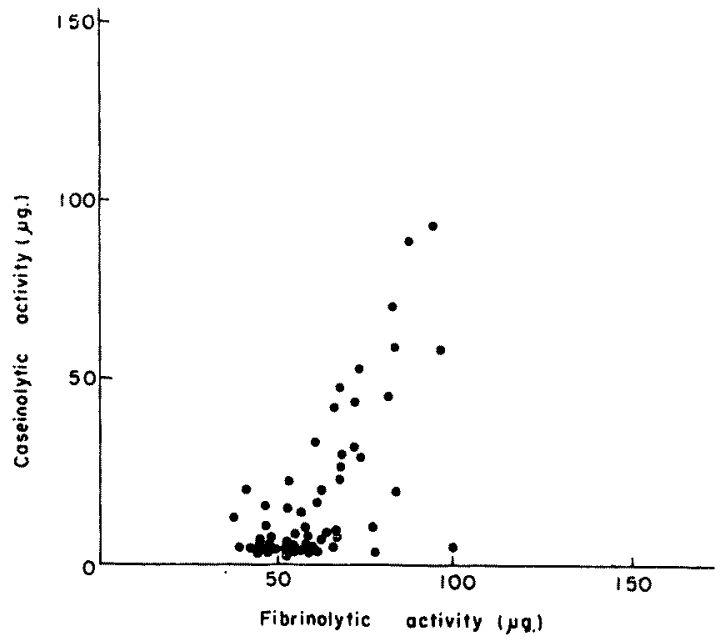

Fig. 2. Relation between spontaneous fibrinolytic and caseinolytic activities of euglobulin fraction.

fibrinolytic activity was found after spinal anesthesia, but the fluctuation of the caseinolytic and TAMe-lytic activities was not remarkable. The fluctuation of the caseinolytic activity, however, was remarkable, especially in patients with postpartum hemorrhage. In particular, high caseinolytic activity at the first stage of delivery decreased rapidly to the normal range after placental expulsion. 
Cancer of cervix

(Radical hysterectomy with lymphnodectomy)

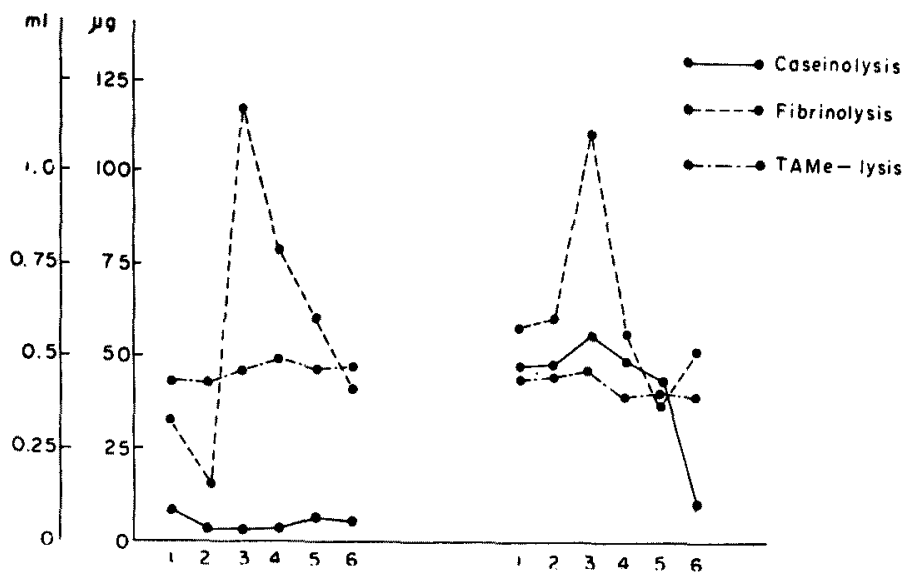

Fig. 3. Changes of spontaneous fibrinolytic, caseinolytic and TAMe-lytic activities during and after radical hysterectomy.

Figures in the abscissa represent the stage of blood collection: 1: before premedication; 2: before spinal anesthesia; 3, 4, and 5: 5, 60 and 120 minutes after spinal anesthesia, 6; 1 day after operation.

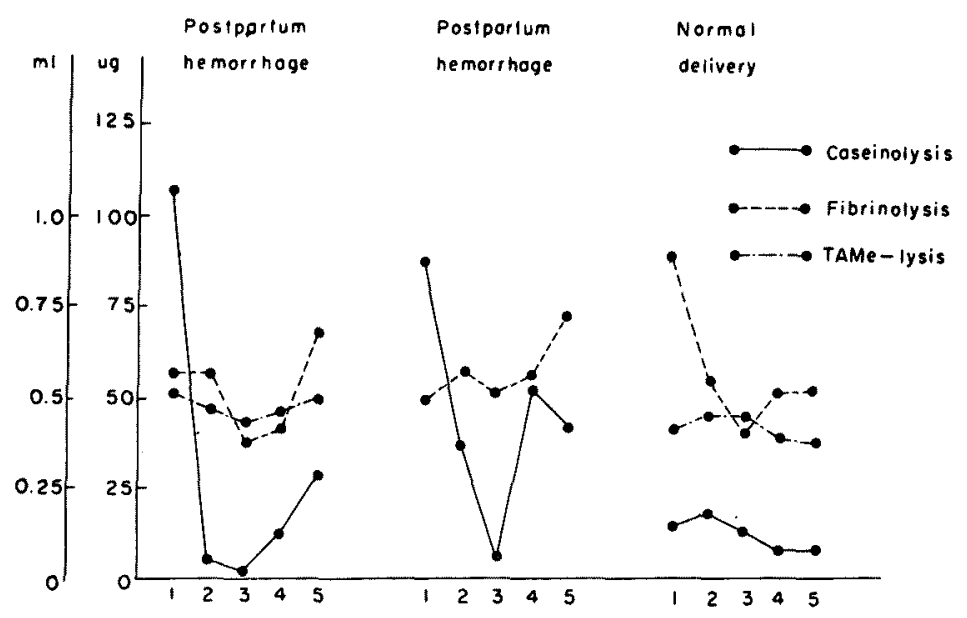

Fig. 4. Changes of spontaneous fibrinolytic, caseinolytic and TAMe-lytic activities during delivery and puerperium.

Figures in the abscissa represent the stage of blood collection: 1: first stage of delivery; 2: immediately after placental expulsion; 3,4 and $5: 1,3$ and 6 days after delivery. 
Furthermore, in two cases of purpura, we had noted that the caseinolytic activity of euglobulin fraction showed a high value before the appearance of purpura. However, the activity decreased to within normal limits when purpura began to appear or become worse. We also noted that the caseinolytic activity of the euglobulin fraction decreased gradually when hemorrhage began in the experimental dog.4) These facts seemed to be very important when one attempts to establish criteria on the results of determined caseinolytic activity of the nagative euglobulin fraction. One must pay attention to the time of collection of the blood sample. If not, there is fear of misleading results. For example, even if the activity of euglobulin obtained from patients who had already hemorrhaged was within normal limits, this value does not always mean a return to physiologic normal.

From the above clinical observations, it can be concluded that the activity of caseinolysis of the euglobulin fraction was not always similar to that of fibrinolysis, and the nature of these two activities seemed to be quite different in some cases.

2. Some evidence suggesting an entity of enzyme multiplicity from the aspect of experimental observations

The chromatograms of protein concentration, caseinolytic, fibrinolytic and TAMe-lytic activities after SK-activation, and antitrypsin activity of the eluates of euglobulin on DEAE-cellulose were summarized in Fig. 5. A relative large protein peak in the eluates of starting buffer and small peaks in those of the other remaining three solvents were observed. The caseinolytic activity was found in concord with protein peaks; however, the activity was nearly equal without close relation to the protein concentration of the eluates. Both fibrinolytic and TAMe-lytic activities were also found corresponding with the protein peaks. The activity of eluates by the final solvent was, however, comparatively low in the fibrinolytic activity and markedly high in the TAMe-lytic activity as compared with other subfractions. Three peaks of the antitrypsin activity were found in the eluates exclusive of the starting buffer. The antitrypsin fractions, which appeared in the second buffer and in the final solvent might also have antiplasmic activity. However, the activity of antiplasmin could not be detected because of the high content of plasminogen in the eluates. The presence of antiplasmic activity in the fraction was detailed in the other paper. ${ }^{5)}$ Thus, the clot-lysis time of eluates by the second buffer and final solvent would be influenced by the antiplasmic activity.

From the above experiments, it would be possible to conclude that there were at least 4 chromatographically different proteolytic enzymes in the euglobulin fraction under these conditions. Especially, the enzyme activity which eluated by the final solvent seemed to be quite different from the other 3 enzymes in the 
Chromatogram of the Euglobulin Fraction on DEAE-cellulose
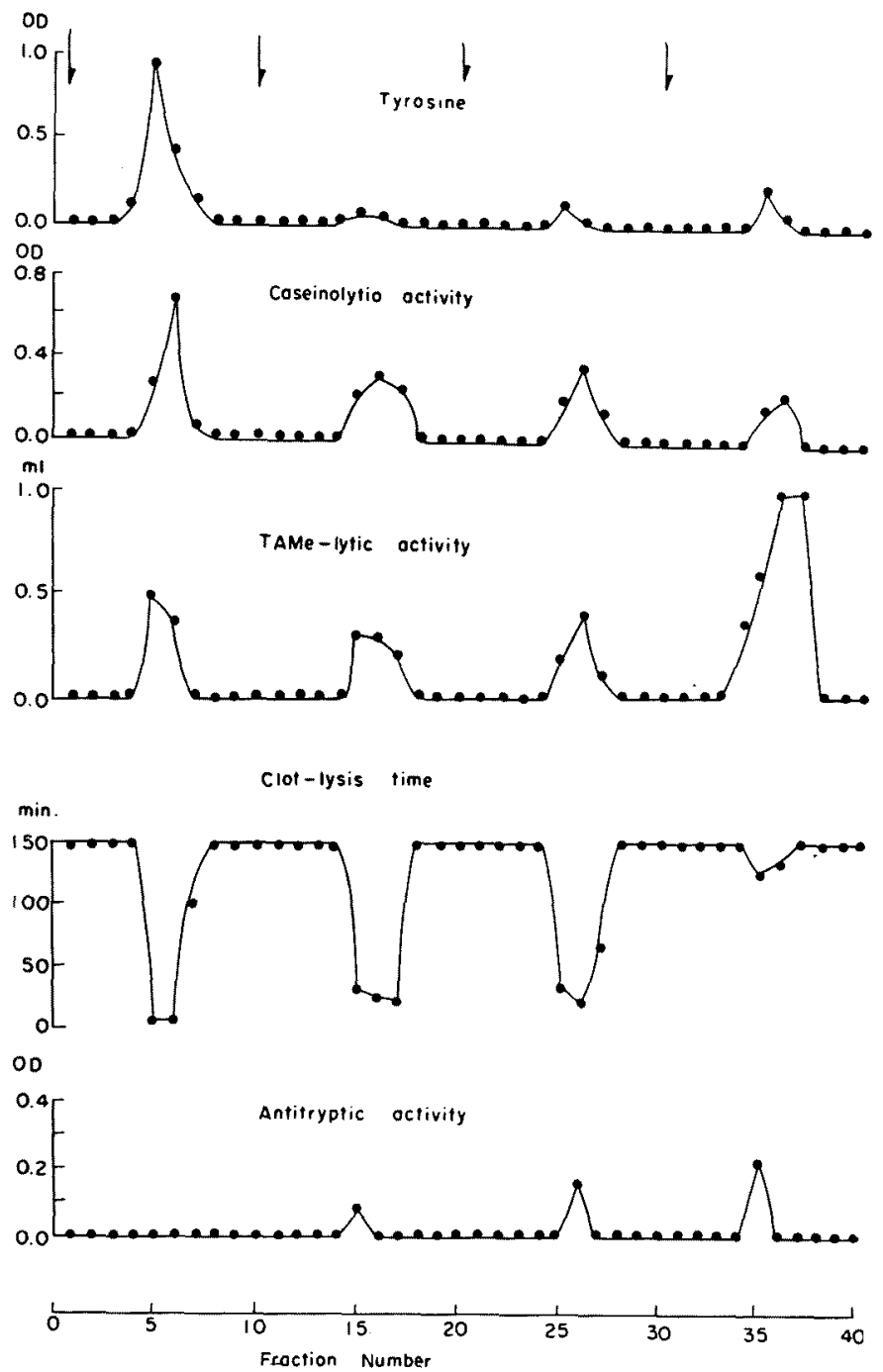

Fig. 5. Chromatogram of protein concentration, caseinolytic, TAMe-lytic, fibrinolytic and antitrypsin activities of subfractionated euglobulin on DEAE-cellulose column.

behavior of its activity of fibrinolysis and TAMe-lysis. However, we could not make ascertion that this multiplicity had not resulted from the secondary changes of the euglobulin under the specified conditions. In addition, the clinical significance of the multiplicity is completely unknown. The recent report of Olow and Nilsson ${ }^{6}$ ) which described the combide use of epsilon aminocaproic acid 
(EACA) and SK for the treatment of thromboembolism was thought to be quite suggestive. They reported that the coagulation defect induced by SK infusion was prevented by supplementary administration of EACA without unduly suppressing the postulated thrombolytic effect. Of course, the action of SK and EACA is quite opposite. In my opinion, the theoretical mechanism of this combined use seemed to be based on the facts that EACA would play a role of inhibiting the fibrinolytic enzyme, and the other different kinds of SK-activatable enzyme would react on the thrombus without general fibrino(geno)lysis in the circulating blood.

In this paper, the possibility of the presence of multiple proteolytic enzymes or isozyme in the euglobulin fraction was preliminarily discussed. For the final conclusion, it should be, of course, required to prepare the pure enzymes in the inhibitor-free state.

\section{SUMMARY}

Some evidences suggesting an entity of proteolytic enzyme multiplicity in the euglobulin fraction were discussed from clinical and experimental observations.

1. The spontaneous caseinolytic activity was not always parallel with the spontaneous fibrinolysis in the euglobulin fraction. A marked fluctuation of the fibrinolytic activity during surgical operations with spinal anesthesia was noted; however, the changes of caseinolysis and TAMe-lysis were unremarkable. On the other hand, the caseinolysis in parturient women with postpartum hemorrhage fluctuated markedly, while the fibrinolysis and TAMe-lysis did not give any remarkable change.

2. There were 4 chromatographically different enzymes demonstrated in the euglobulin fraction on DEAE-cellulose column chromatography.

\section{References}

1) Okamoto, S., Personal communication.

2) Maki, M., Tohoku J. Exper. Med., 1962, 78, 264.

3) Troll, S., Sherry, S. \& Wachman, J., J. Biol. Chem., 1954, 208, 85.

4) Kikuchi, E., Kikuchi, I. \& Maki, M., Jap. J. Obst. \& Gynec., 1961, 13, 1092.

5) Maki, M., ibid., 1963, 15. (2) press.

6) Olow, B. \& Nilsson, I.M., Acta Chirurg. Scand., 1962, 124. - translated by Brown, J.L., Malmoe, 1962, Lundgrens Soener Boktryckeri. 